\title{
Analytical Analysis and Experimental Validation of a Multi-parameter Mach- Zehnder Fiber Optic Interferometric Sensor
}

\author{
Lorenzo B. Scandian, Marcelo E. V. Segatto, Carlos E. S. Castellani \\ Telecommunications Laboratory (LABTEL), Electrical Engineering Department, Federal University of Espirito \\ Santo, Vitória-ES 29075-910, Brazil \\ lorenzo.scandian@outlook.com \\ segatto@ele.ufes.br \\ carlos.castellani@ufes.br
}

\begin{abstract}
Here we report a simple analytical technique to model a Mach-Zehnder fiber optic interferometric sensors that allow us to predict and calculate via computer simulations parameters that are not easily obtained experimentally. This model was calibrated and compared with experimental data using a $120 \mathrm{~mm}$ sensor for measurements of temperature, refractive index and water level. For instance, we were able to calculate the effects on the cladding effective index caused by the variation of those physical parameters. Moreover, this analysis could further our understanding of such sensors and allow us to make predictions about their use in different applications and even their behavior with different sensing lengths.
\end{abstract}

Index Terms - Optical fiber interference, optical interferometry, optical fiber applications, optical fiber devices, analytical model.

\section{INTRODUCTION}

The use of fiber optic sensors is in considerable growth. Their applications range from structural health to ecological monitoring. Since the last decades, these sensors have been widely used in industries due to their enormous advantages such as high sensitivity, precision, immunity to electromagnetic fields, and safe implementation and operation. Within range of optical sensors, interferometric techniques stand out for usually allowing greater sensitivity than traditional ones. Measurements of liquid level, [1]-[3], temperature, [1], refractive index, [1], [4], and angles can be very accurate when performed by optical interferometric sensors.

Several interferometric sensors have been published based on the traditional principles of MachZehnder, [1], [3], [5]-[7]. It has been widely seen that for each sensor constructed, values such as their sensitivity and operational range is often highly dependent on the sensors length. This means that to find an analytical or numerical model that can accurately describe the functioning of a specific type of sensor is imperative in order to appropriately project new sensors with certain characteristics. Amongst the Mach-Zehnder optical fiber sensors category a very common type are the sensors where their functioning rely on the principle that part of the incident radiation is coupled to the fiber cladding and allowing variations of the external medium to cause variations on the cladding effective refractive index $\left(\Delta n_{e f f}\right)$. Those variations can be tracked by analyzing the interference pattern 
obtained when summing the radiation coming from both the core and the cladding, variations which most of times are translated to a shift in wavelength of a specific spectral peak or valley. Because of this behavior, the actual variation of the $\Delta n_{e f f}$ as a response to variations of the external medium parameters usually remains unknown. Even though, a precise knowledge and calculation of this variation is often not needed for the sensor to function, it can be very useful to have a more complete understand of the behavior of the sensor which can be used to improve existing devices and also to predict and project new types of sensors with specific characteristics.

This paper presents the usage of an analytical description to perform simulations of a specific type of sensors through a simple model developed based, calibrated and validated on the experimentally demonstrated case of a Mach-Zehnder multi-parameter interferometric sensor with $120 \mathrm{~mm}$ length [1]. This analytic model allows predicting via computer simulations precisely how much external variations of temperature, refractive index and liquid level cause changes on the cladding refractive index. This can, for instance, allow us to predict the sensitivities that would be obtained for smaller sensors and also project the use of such devices to split pulses in time and to delay them with tunability depending on an external physical parameter such as the liquid level. The results here obtained can potentially be extended to any similar Mach-Zehnder type optical fiber interferometer.

\section{NUMERICAL MODELING}

Interferometric sensors such as [1], [3]-[5] use interrogation techniques based on the spectrum obtained by the interference of light beams propagating inside the optical fiber through different routes. The idea is to give the light two paths; one being influenced by the external environment while the other is not. Thus, at the receiving end of the light both signals are summed and the variations of physical parameters of the external medium are analyzed by the resulting spectra. Here we choose the sensor experimentally demonstrated in [1] to construct this analytic model, which separates both optical paths by adding an axial offset in the splicing between two fibers with different core diameters that compose the device. The first fiber is a standard telecom fiber (STF) with $10 \mu \mathrm{m}$ diameter and the second is a $12 \mathrm{~cm}$ single mode fiber with $6 \mu \mathrm{m}$ diameter (Raman OFS). This offset splice and the difference in core diameters makes part of the incident light to couple to the cladding, which is later recombined to the part propagating in the core by the use of a multi-mode fiber of $50 \mu \mathrm{m}$ diameter spliced without axial off-set to the end of the sensor, as shown in Fig.1. Nevertheless, it is important to note that our simulations could easily be adapted and calibrated based on Mach-Zehnder fiber sensors build by other techniques.

Based on this light scattering event, we can model the described behavior by simply adding two electric fields, $E_{1}$ e $E_{2}$, representing the light beams in the core and cladding, respectively. These electric fields can be mathematically defined by (1) and (2), where $\omega$ is the light beam angular frequency in rad/s, $A_{1}$ and $A_{2}$ are the amplitudes of the respective electric fields, $z$ is the length of the waveguide, in this case, the length of the sensor, and $\beta_{1}$ and $\beta_{2}$ are the wavenumber of the core and 
cladding respectively.

$$
\begin{aligned}
& E_{1}=A_{1} \sin \left(\omega t-\beta_{1} z\right) \\
& E_{2}=A_{2} \sin \left(\omega t-\beta_{2} z\right)
\end{aligned}
$$

a)

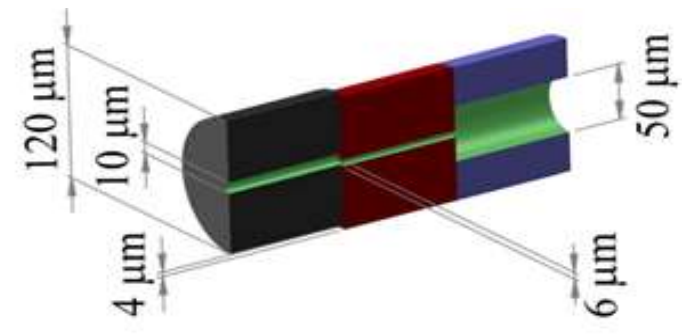

b)

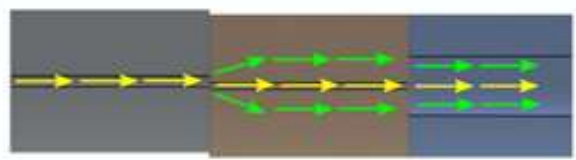

c)

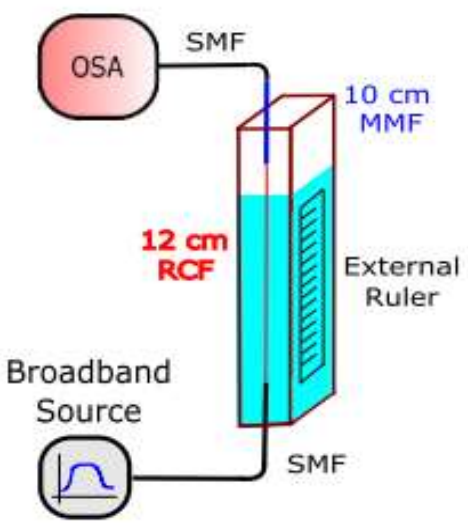

Fig. 1. Fiber arrangement using an off-setted fiber with reduced core to generate the cladding modes. a) 3-D view, b) longitudinal section, c) sensor schematic used in the experiments.

The wavenumbers $\beta_{1}$ and $\beta_{2}$ are values that depend on the beams wavelengths $\lambda_{1}$ e $\lambda_{2}$ as defined by (3) and (4), which are functions of the refractive index of the core and cladding, respectively. The method used to relate $\beta$ with the refractive index $n$, is according to the already known (5) and (6), where $v, f$ and $c$ represent the wave propagation speed, frequency, and speed of light in vacuum, respectively.

$$
\begin{aligned}
& \beta_{1}=\frac{2 \pi}{\lambda_{1}} \\
& \beta_{2}=\frac{2 \pi}{\lambda_{2}} \\
& v=\lambda f
\end{aligned}
$$




$$
n=\frac{c}{v}
$$

The interrogation performed in [1] is based on the shift in wavelength of peaks and valleys generated in the spectrum after the combination of both signals traveling in the cladding and core. This happens because when the external medium characteristics are changed, the effective refractive index of the cladding is changed, altering the value of $\beta_{2}$ but maintaining constant the value of $\beta_{1}$. Thus, from analyzing the experimental data obtained in [1], we can calculate precisely what is the corresponding change of the cladding effective refractive index as a function of the external medium liquid level, temperature, and its refractive index. In order to simulate the broadband optical source used in [1] as the sensor input to generate the spectrum obtained after the sensor when combining both beams, we use as input different wavelengths for the electric fields $E_{1}$ and $E_{2}$ ranging from $1500 \mathrm{~nm}$ to $1580 \mathrm{~nm}$. Using the parameters defined in [1] of $\mathrm{z}=12 \mathrm{~cm}$, core refractive index $\left(n_{1}\right)=1.4565$ and cladding refractive index $\left(n_{2}\right)=1.45$ we can obtain the optical spectrum shown in Fig. 2.

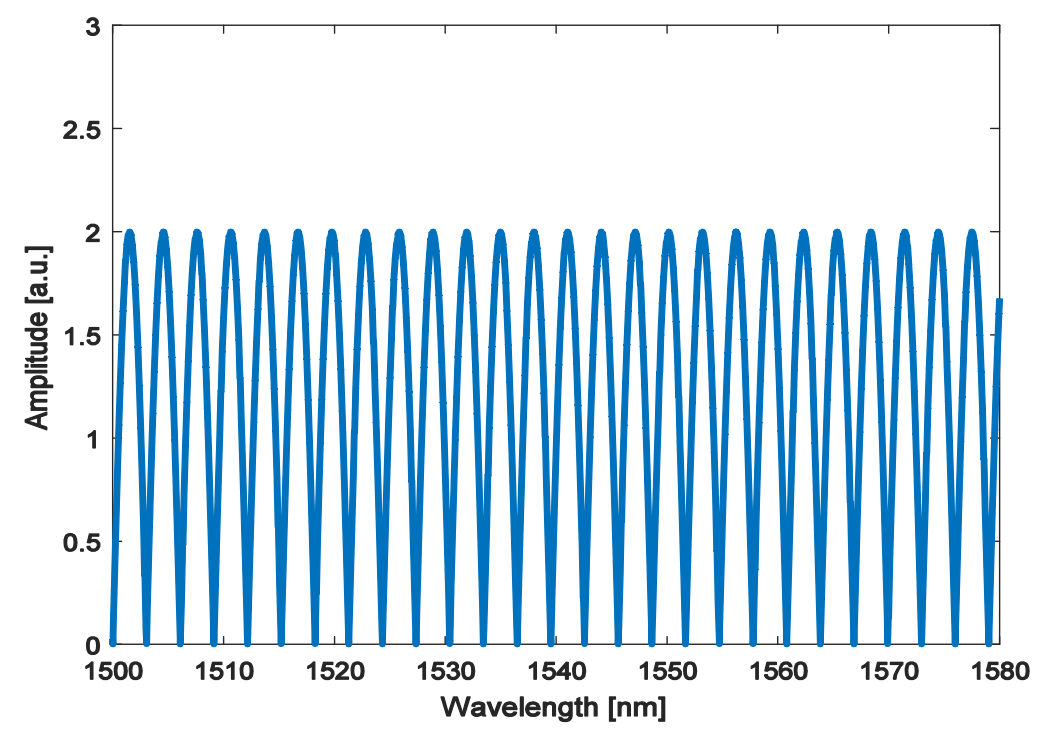

Fig. 2. Analytic interferometric pattern.

As already mentioned, the studied sensor uses the variation of the cladding effective refractive index, which results in the displacement of peaks and valleys of the interferometric spectrum, in order to perform its measurements. Thus, in our simulations we vary the effective refractive index of the cladding $\left(n_{2}\right)$ and we compare the obtained wavelength shift of a given peak with the data obtained from [1] as a function of the external medium level, temperature and refractive index.

\section{RESULTS}

\section{A. Liquid's Level Sensing Simulation}

According to the performed calibration, the initial cladding refractive index that correctly feats the peaks obtained from the simulation with the experimental data is $n_{2}=1.4500023$. In order to reach the wavelength variation of the peak initially centered in $1541.05 \mathrm{~nm}$, as it was chosen in [1], that occurs when filling of the container with $120 \mathrm{~mm}$ of water, the cladding effective refractive index was Brazilian Microwave and Optoelectronics Society-SBMO received 29 Sept 2018; for review 5 Nov 2018; accepted 13 Nov 2018 
varied until the final value of $n_{2}=1.4499992$. The simulation was done for steps of the cladding effective refractive index of $10^{-7}$ refractive index units (RIU), resulting in 31 points and a negative total change of $\Delta n_{\text {eff }}=-3.1 \times 10^{-6}$ RIU. Each interaction leads to a spectral shift to the left, as it was also seen in [1]. Fig. 3 shows the simulation spectra obtained for the peak initially centered at $1541.05 \mathrm{~nm}$ as function of the water level. As it can be seen, when the sensor is completely filled with water $(120 \mathrm{~mm})$ the central wavelength shifts to $1540.32 \mathrm{~nm}$ which corresponds to a variation of the cladding effective index of $-3.1 \times 10^{-6}$ RIU.

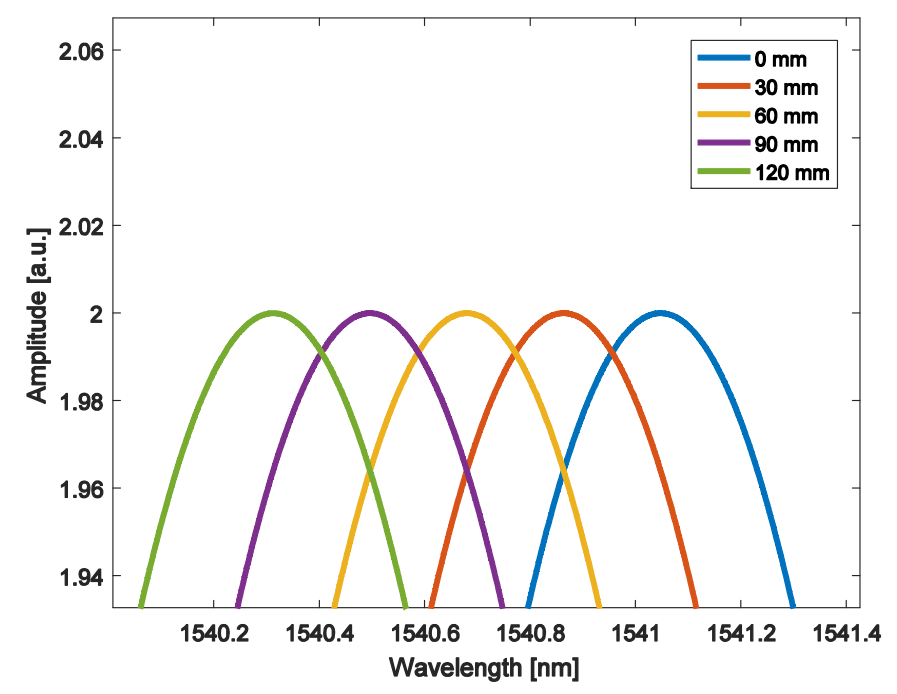

Fig. 3. Spectrum corresponding of the peak initially centered at $1541.05 \mathrm{~nm}$ as a function of water level.

From the successive positions of the displaced peak shown in Fig. 3, we were able to compare our simulation results with the experimental data measurements, which is shown in Fig. 4, where the central wavelength of the peak initially centered is plotted against the water level variation.

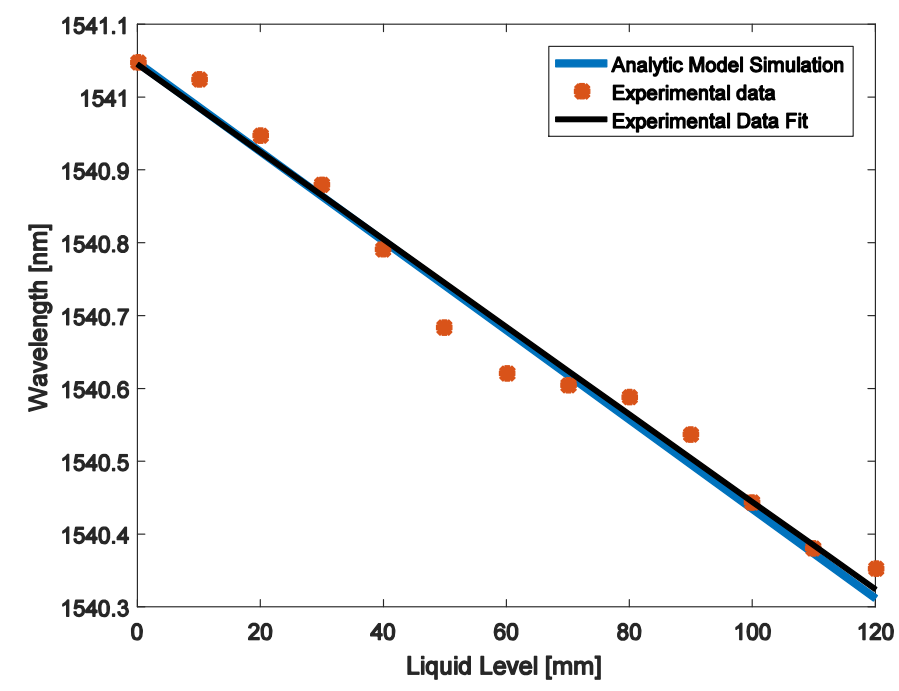

Fig. 4. Central wavelength of the peak initially at $1541.05 \mathrm{~nm}$ as a function of the liquid level.

This result highlights the fact that our simple approach of modeling our sensor only as a sum of two different optical paths can give a very accurate prediction of the sensor behavior, avoiding the Brazilian Microwave and Optoelectronics Society-SBMO received 29 Sept 2018; for review 5 Nov 2018; accepted 13 Nov 2018 Brazilian Society of Electromagnetism-SBMag 
necessity of performing a more complete modal analysis which is much more time consuming and computationally complex.

Finally, in Fig. 5 it can be seen how the variation of water level affects the actual effective refractive index of the cladding. It can be observed the variation is linear with a rate of $-2.58 \times 10^{-8}$ $\mathrm{RIU} / \mathrm{mm}$. It is important to note that an increase in liquid level actually decreases the effective refractive index of the cladding. Moreover, it is clear that even such small variation in effective refractive index of $-2.58 \times 10^{-8} \mathrm{RIU} / \mathrm{mm}$ can give rise to a moderate sensor sensitive when analyzing in terms of wavelength shift, which is $6 \mathrm{pm} / \mathrm{mm}$.

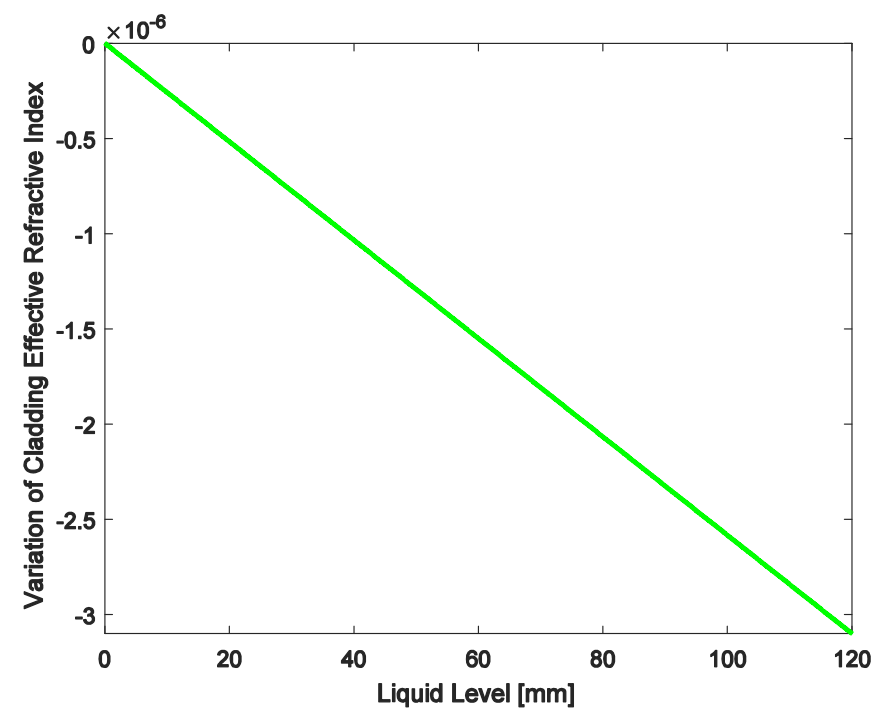

Fig. 5. Variation of cladding effective refractive index as a function of the liquid level.

\section{B. Liquid Temperature Sensing Simulation}

Analogous to the liquid level measuring, for temperature sensing simulation we used the same method already described. Nevertheless, some points in this simulation should be highlighted. To measure the temperature, the experimental data [1] varied the temperature of the water with the pipette where the sensor was placed with the $120 \mathrm{~mm}$ completely full. This means that the initial cladding effective refractive index is not the same as the one in the previous topic. For this procedure, we calibrated the refractive index to have the same value of $n_{1}=1.4565$ but a value $n_{2}=1.4499987$. Note that this value is very close to the one found for the filled vessel in the liquid level sensing simulation. The difference of $5 \times 10^{-7} \mathrm{RIU}$ is attributed to the fact that the level experiment was done when the temperature was $25^{\circ} \mathrm{C}$ degrees and here the simulation and the temperature experimental data variations start at $22^{\circ} \mathrm{C}$.

In order to achieve the wavelength variation that occurs according to the liquid temperature change from $22^{\circ} \mathrm{C}$ to $55^{\circ} \mathrm{C}$, the cladding effective refractive index was varied to the final value of $n_{2}=$ 1.4500043. As in the previous topic, the value of each step for the variation of the effective refractive index was $10^{-7}$ RIU, resulting in 58 interactions and a positive variation of $\Delta n_{e f}=5.6 \times 10^{-6}$ RIU. Due to the positive variation, this time the spectral shift occurred to the right. 
The central wavelength of the peak initially centered at $1540.2 \mathrm{~nm}$ as a function of temperature can be seen in Fig. 6. Again we see that our simplified approach of modeling the sensor as only the sum of two electric fields traveling through two different optical paths describes the sensor behavior very accurately. The variation of the cladding refractive index as a function of temperature is shown in Fig. 7, where again we obtain a linear relation, but now presenting a positive inclination and a sensitivity of $1.697 \times 10^{-7} \mathrm{RIU} /{ }^{\circ} \mathrm{C}$. This positive inclination in Fig. 7 means that increasing the water temperature caused an increase in effective refractive index of the cladding, which leads to a red shift in the central peak wavelength as it was obtained also experimentally. Again, it can be observed that even a very small sensitivity of $1.697 \times 10^{-7} \mathrm{RIU} /{ }^{\circ} \mathrm{C}$ can lead in terms of spectra wavelength variation to a moderately high sensor sensitivity of $39 \mathrm{pm} /{ }^{\circ} \mathrm{C}$.

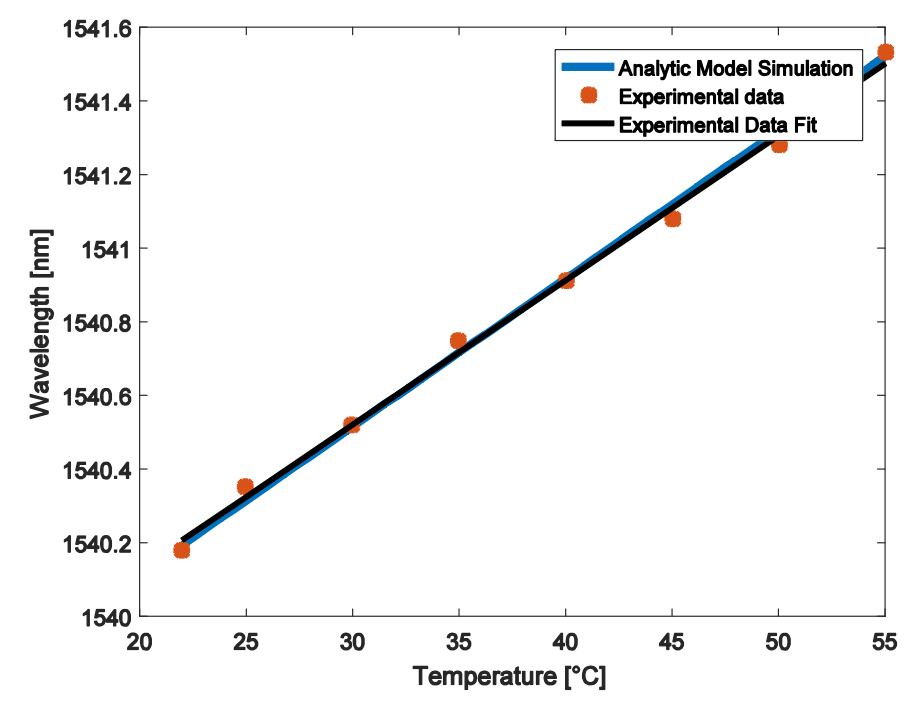

Fig. 6. Central wavelength of the same peak as a function of the temperature.

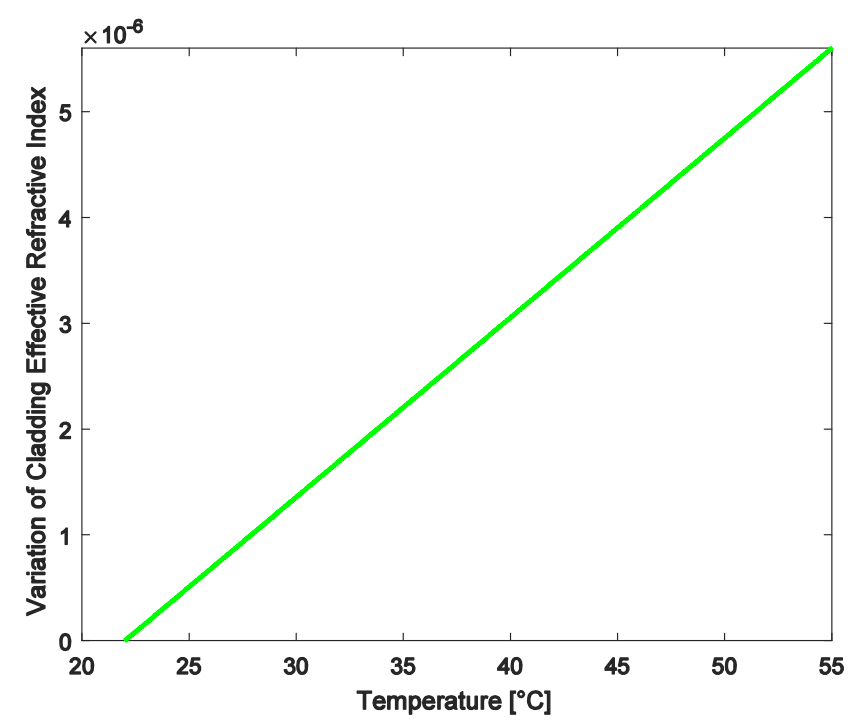

Fig. 7. Variation of cladding effective refractive index as a function of liquid temperature. 


\section{Liquid Refractive Index Sensing Simulation}

Using the experimental data to calibrate the liquid refractive index sensing simulation we found that the best cladding effective refractive index to start with was $n_{2}=1.4500001$, which is due to the fact that the experimental procedure [1] used a $60 \mathrm{~mm}$ full pipette because it presented the highest level of linearity. In the experiment the external refractive index was varied by adding glycerin to the water surrounding the sensor. As it happened for the last two cases, in order to reach the spectral wavelength variation that was obtained experimentally for a liquid refractive index change from 1.333 to 1.442 , the cladding effective refractive index was varied until the value $n_{2}=1.4499961$. As in all previous topics, the value of each step of the variation of the effective refractive index was $10^{-7} \mathrm{RIU}$, resulting in 40 interactions and a negative variation of $\Delta n_{e f f}=-4.0 \times 10^{-6}$ RIU.

The central wavelength of the peak initially centered at $1540.6 \mathrm{~nm}$ as a function of the refractive index of the external medium is shown in Fig. 8. In this case, as it happened for the water level measurements, the spectral shift occurred to the left as the refractive index increases. Moreover, again it can be seen the accuracy in which our simple analytical model suits for modeling precisely the experimental data. In Fig. 9 we can see the effective cladding refractive index as a function of the refractive index of the external medium. It is possible to observe that again the relation is linear with a negative inclination of $-36.67 \times 10^{-6} \mathrm{RIU}_{\text {cladding }} / \mathrm{RIU}_{\text {external. }}$. Additionally, this small sensitivity of $-36.67 \times 10^{-6} \mathrm{RIU}_{\text {cladding }} / \mathrm{RIU}_{\text {external }}$ allows the creation of an optical sensor in terms of wavelength shift with a high sensitivity of $8.8 \mathrm{~nm} / \mathrm{RIU}$.

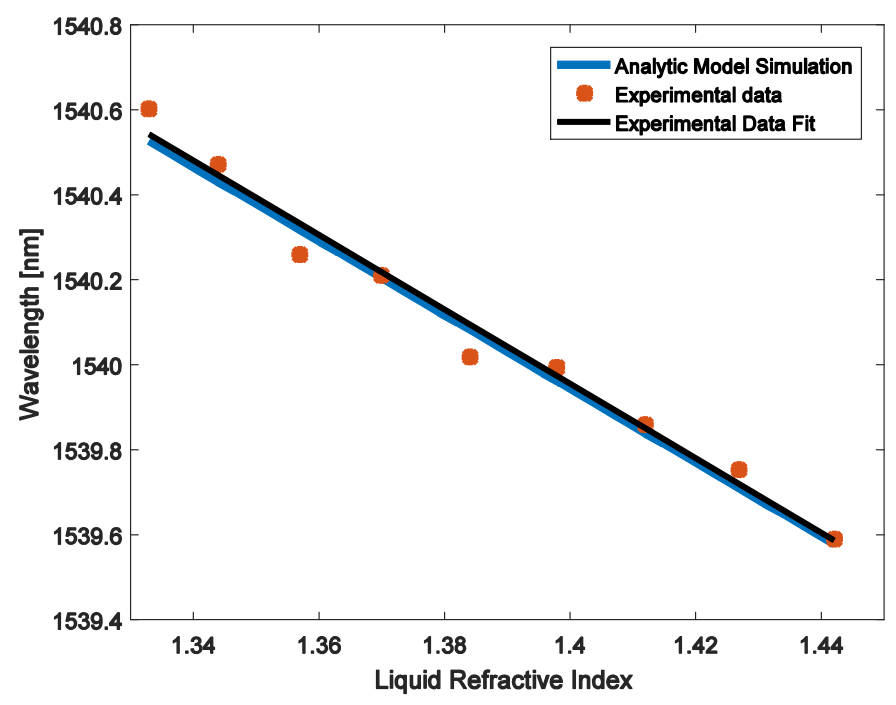

Fig. 8. Central wavelength of the same peak as a function of liquid refractive index. 


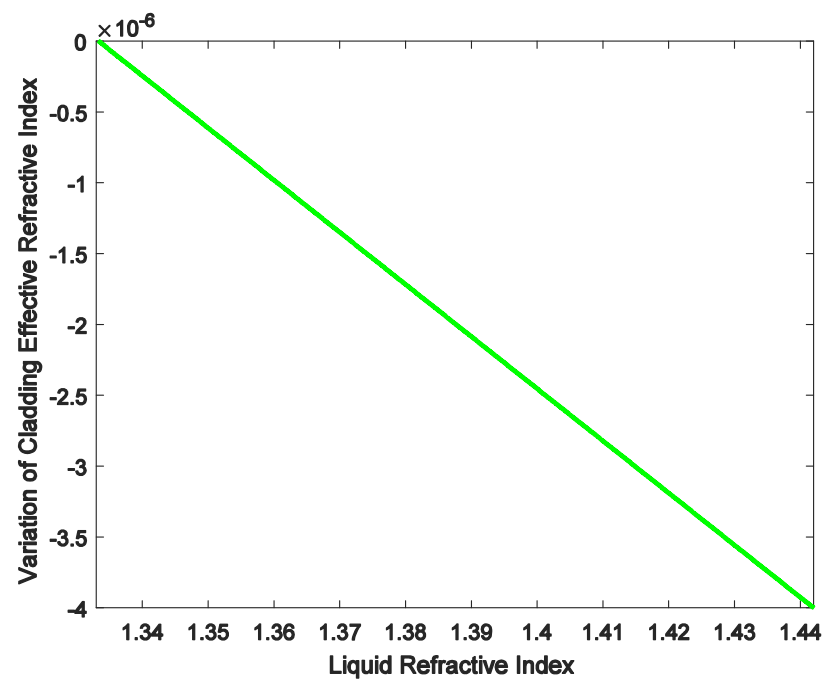

Fig. 9. Variation of cladding effective refractive index as a function of the liquid refractive index.

\section{Sensor length variation analysis}

In this section we used the analytical model to investigate the behavior of the sensor for variations of the sensor length. Initially, the effective refractive index was set to its initial value in the absence of water and the sensor size varied from $1 \mathrm{~cm}$ to $12 \mathrm{~cm}$. Fig. 10 shows the spectrum obtained for sensors with the lengths of $4 \mathrm{~cm}, 8 \mathrm{~cm}$ and $12 \mathrm{~cm}$. As it can be seen, as the sensor becomes shorter the bandwidth of each peak increases, and that also increases the distance between consecutive peaks, or the free spectral range (FSR). The initial position of each peak also varies when changing the sensor length.

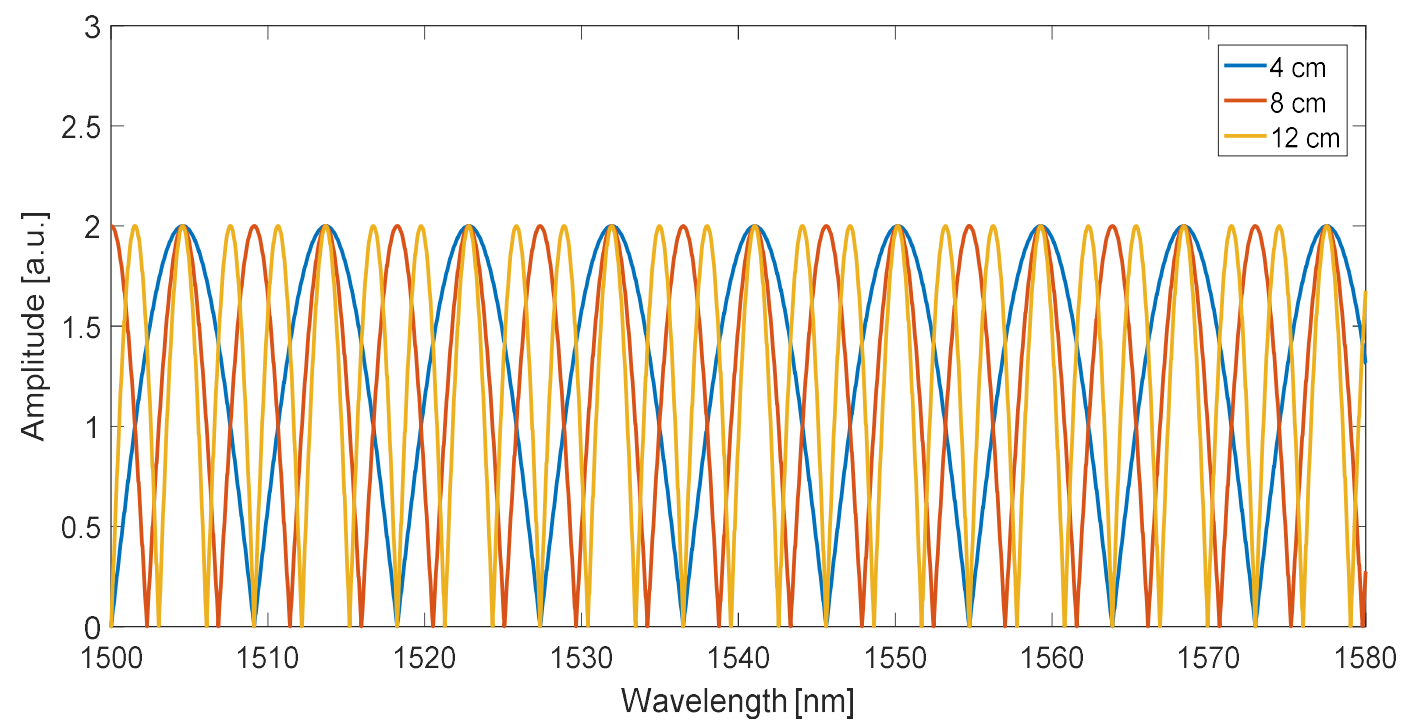

Fig. 10. Spectra obtained for sensor of $4 \mathrm{~cm}, 8 \mathrm{~cm}$ and $12 \mathrm{~cm}$ simulating the scenario without water.

The free spectral range as a function of the sensor length can be seen in Fig. 11. As expected, we can observe an exponential decay, which is a typical feature of a Mach-Zehnder interferometer. For instance, interferometers with FSRs bigger than a nanometer could be obtained for sensors shorter 
than $5 \mathrm{~cm}$, which would allow a very big visualization window around each peak to analyze its variation as a function of external physical parameters. However, this result shows that the use of sensors that are longer than a few tens of centimeters as optical sensing devices can be problematic since a small FSR means that consecutive peaks are very close together, which would make necessary the use of a very small wavelength window around a given peak to analyze its variation.

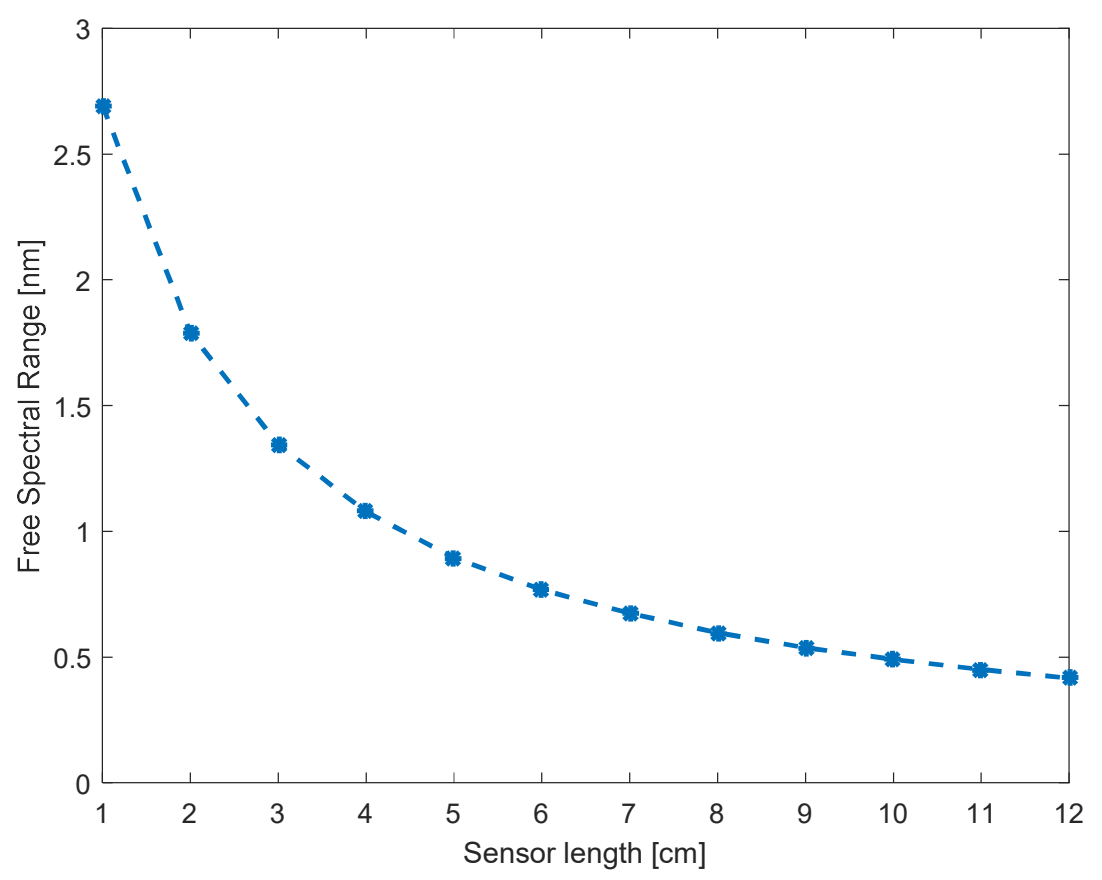

Fig. 11. Free spectral range as a function of the sensor length.

Finally, again for sensor lengths from 1 to $12 \mathrm{~cm}$, the effective refractive index of the cladding was varied simulating exactly the changes in water level, temperature and refractive indexes used in the previous sections. The sensitivity of the peak closest to $1541 \mathrm{~nm}$ for variations of water level obtained as a function of the sensor length can be seen in Fig. 12. It was observed that the sensitivity of the sensor remains constant, with the value of $6 \mathrm{pm} / \mathrm{nm}$ regardless of the sensor length chosen. Similar results were obtained when analyzing the sensor sensitivity to temperature and refractive index, that also remained constant with the values of $39 \mathrm{pm} /{ }^{\circ} \mathrm{C}$ and $8.8 \mathrm{~nm} / \mathrm{RIU}$, respectively, for any given sensor length. Nevertheless, it is important to highlight the fact that, our analytical analysis is based on a simplified model considering only one average cladding mode. Therefore, sensors experimentally built with other lengths may exhibit sensitivity variations due to factors that are not addressed in this model, such as the fact that some cladding modes might propagate in the fiber for longer distances then others, being relevant only for sensors up to a certain length. 


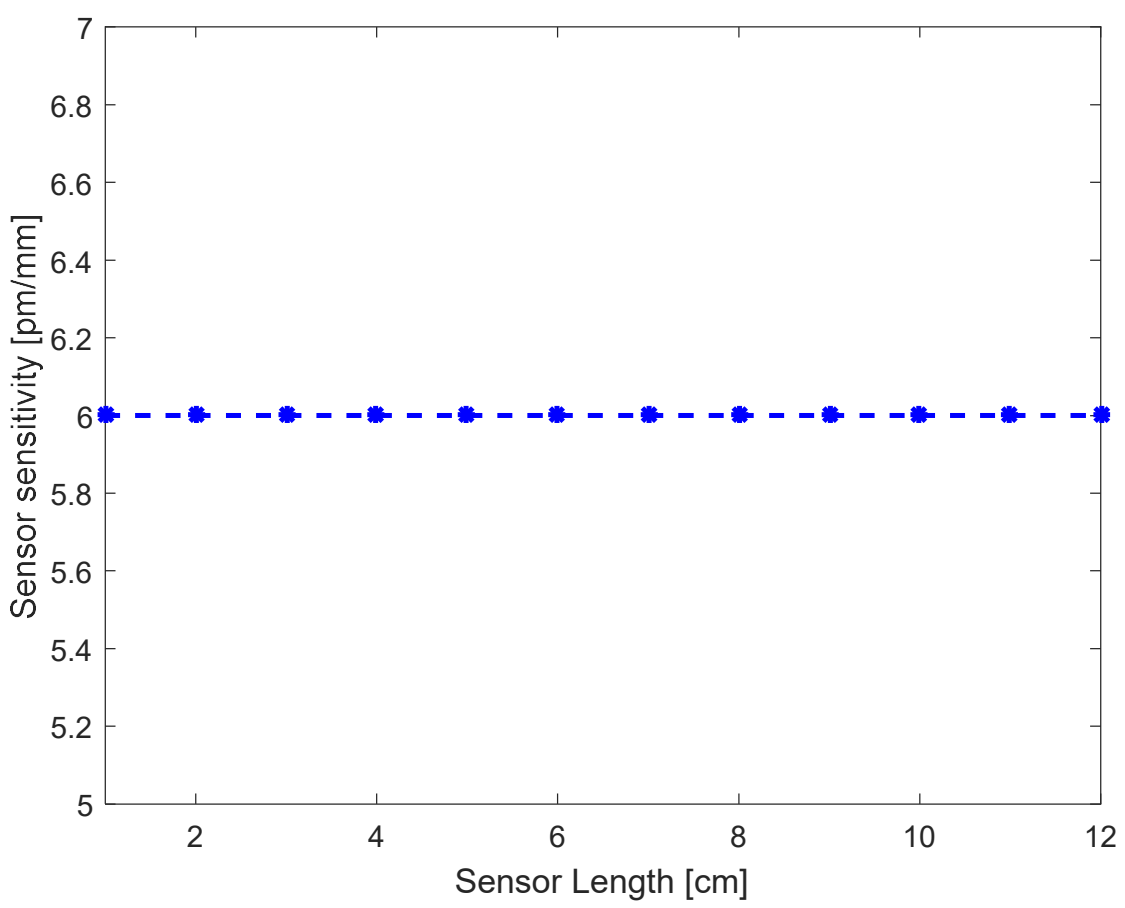

Fig. 12. Sensor sensitivity for water level variations as a function of the sensor length.

\section{CONCLUSION}

In conclusion, we presented a simple analytical model that describes the behavior of an all-fiber optic interferometric sensor that allow us to simulate its usage for liquid level sensing, liquid temperature sensing, and liquid refractive index sensing. In this paper, we are also able to formulate a deeper understanding of how those external physical parameters affect the cladding effective refractive index, showing for each case its sensitivity and variation rate. This simple analytical formulation was compared and validated with experimental data showing that more complete modal analysis is not needed in order to understand and predict the functioning of such sensors. This understanding can be applied to project similar sensors of other lengths and also to simulate the use of a similar structure for other applications. Those results could potentially be extended to many other Mach-Zehnder type fiber optical sensor.

\section{REFERENCES}

[1] C. Castellani, H. Ximenes, R. Silva, A. Frizera-Neto, M. Ribeiro, M. J. Pontes, "Multi-Parameter Interferometric Sensor Based on a Reduced Diameter Core Axial Offseted Fiber," IEEE Photonics Technology Letters, vol. 29, no. 2, pp. 239-242, January 2017.

[2] H. Gong, H. Song, S. Zhang, K. Ni, and X. Dong, "An optical liquid level sensor based on polarization-maintaining fiber modal interferometer," Sens. Actuators A, Phys., vol. 205, pp. 204-207, Jan. 2014.

[3] C. A. Rodriguez, M. R. N. Ribeiro, A. Frizera-Neto, C. E. S. Castellani, and M. J. Pontes, "Envelope-based technique for liquid level sensors using an in-line fiber Mach-Zehnder interferometer," Appl. Opt., vol. 55,pp. 9803-9809, 2016.

[4] X. Wen, T. Ning, H. You, J. Li, T. Feng, L. Pei, and W. Jian, "DumbbellShaped MachZehnder Interferometer With High Sensitivity of Refractive Index," in IEEE Photonics Technology Letters, vol. 25, pp. 1839-1842, 2013.

[5] Z. Li, C. Liao, Y. Wang, L. Xu, D. Wang, X. Dong, S. Liu, Q. Wang, K. Yang, and J. Zhou, "Highly-sensitive gas pressure sensor using twin-core fiber based in-line Mach-Zehnder interferometer," Opt. Express, vol. 23, pp. 66736678,2015

[6] L. Li, X. Li, Z. Xie, and D. Liu, “All-fiber Mach-Zehnder interferometers for sensing applications,” Opt. Exp., vol. 20, no. 10, pp. 11109-11120, May 2012.

[7] J. Chen, J. Zhou, and Z. Jia, "High-sensitivity displacement sensor based on a bent fiber Mach-Zehnder interferometer," IEEE Photon. Technol. Lett., vol. 25, no. 23, pp. 2354-2357, Dec. 1, 2013. 